\title{
Top-quark physics at CLIC
}

\author{
Aleksander Filip Żarnecki ${ }^{* \dagger}$ on behalf of the CLICdp Collaboration \\ Faculty of Physics, University of Warsaw \\ E-mail: Filip.Zarnecki@fuw.edu.pl
}

The Compact Linear Collider (CLIC) is a mature option for a future electron-positron collider operating at centre-of-mass energies of up to $3 \mathrm{TeV}$. CLIC will be built and operated in a staged approach with three centre-of-mass energy stages currently assumed to be $380 \mathrm{GeV}, 1.5 \mathrm{TeV}$ and $3 \mathrm{TeV}$. This contribution discusses the prospects for precision measurements of top-quark production and properties at CLIC. Based on a dedicated centre-of-mass energy scan around the topquark pair production threshold, the top-quark mass can be determined with a precision of about $50 \mathrm{MeV}$ in a theoretically well-defined manner. Other approaches to extract the top-quark mass at the first stage of CLIC make use of ISR photons or the direct reconstruction of the top quarks. The large number of top-quark pairs produced also allows competitive searches for Flavour Changing Neutral Current (FCNC) top-quark decays to charm quarks. At the high-energy stages of CLIC the top Yukawa coupling and $\mathrm{CP}$ properties in the $\mathrm{ttH}$ coupling can be studied, as well as the top-quark pair production through the vector-boson fusion. Expected limits on new physics contributions described in terms of Effective Field Theory (EFT) operator coefficients are presented, showing sensitivity of CLIC to mass scales up to $100 \mathrm{TeV}$.

European Physical Society Conference on High Energy Physics - EPS-HEP2019 -

10-17 July, 2019

Ghent, Belgium

\footnotetext{
* Speaker.

${ }^{\dagger}$ Supported by the National Science Centre, Poland, project nr 2017/25/B/ST2/00496 (2018-2021).
} 


\section{Introduction}

The Compact Linear Collider (CLIC) is a mature option for a future electron-positron collider operating at centre-of-mass energies of up to $3 \mathrm{TeV}[1,2]$. Top-quark physics, together with Higgs boson studies and searches for Beyond the Standard Model (BSM) phenomena, is assumed to be one of the main pillars of the CLIC research program. Processes involving top quarks provide unique opportunities to test the Standard Model (SM) predictions and look for possible signatures of new physics beyond the SM (BSM). This contribution summarizes prospects for precision measurements of top-quark properties and resulting BSM constraints presented by the CLIC Detector and Physics collaboration (CLICdp) [3]. These results are based on detailed simulation studies, taking into account luminosity spectra and beam induced backgrounds, full detector simulation based on GEANT4, final state reconstruction based on the particle flow approach with PANDORAPFA, jet clustering with the VLC algorithm as implemented in the FASTJET package and flavour tagging with LCFIPLUS.

\section{Top-quark mass measurements}

A dedicated scan of the top-quark pair production threshold is expected to be the most precise method to determine of the top-quark mass, both in terms of the statistical and of the systematic uncertainties. The baseline CLIC running scenario [4] assumes that an integrated luminosity of $100 \mathrm{fb}^{-1}$ will be collected at the top pair production threshold in ten equidistant points, with $10 \mathrm{fb}^{-1}$ per point and a point-to-point spacing of $1 \mathrm{GeV}$, as illustrated in Fig.1 (left). For the two dimensional mass and width fit to the threshold scan data, the expected statistical uncertainty on the top-quark mass of about $20 \mathrm{MeV}$ is obtained, assuming a dedicated (reduced charge) luminos-
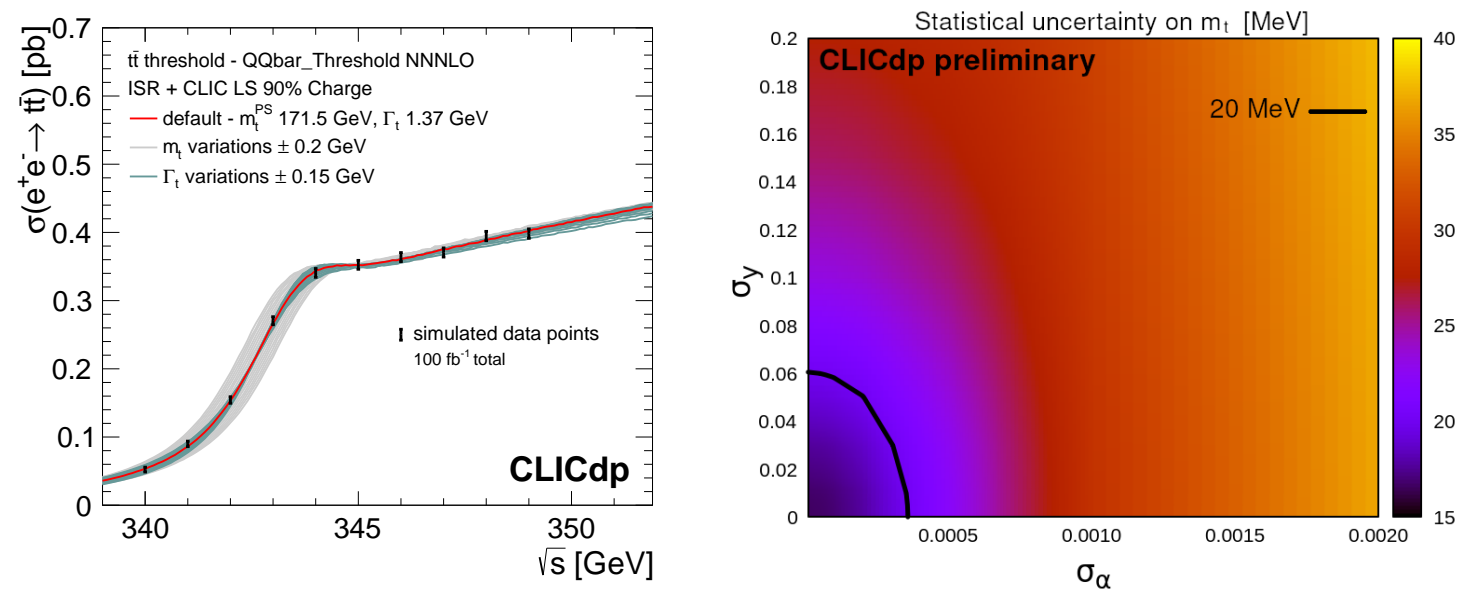

Figure 1: Top-quark threshold scan at CLIC with a total integrated luminosity of $100 \mathrm{fb}^{-1}$ for running with a dedicated (reduced charge) luminosity spectrum; the bands around the central cross section curve indicate the dependence of the cross section on the top-quark mass and width (left) [3]. Expected statistical uncertainty on the top-quark mass from the fit involving all considered model parameters, as a function of the strong coupling constant uncertainty, $\sigma_{\alpha}$, and top Yukawa coupling uncertainty, $\sigma_{y}$, from independent measurements, assuming normalisation uncertainty of $0.1 \%$ (right) [5]. 

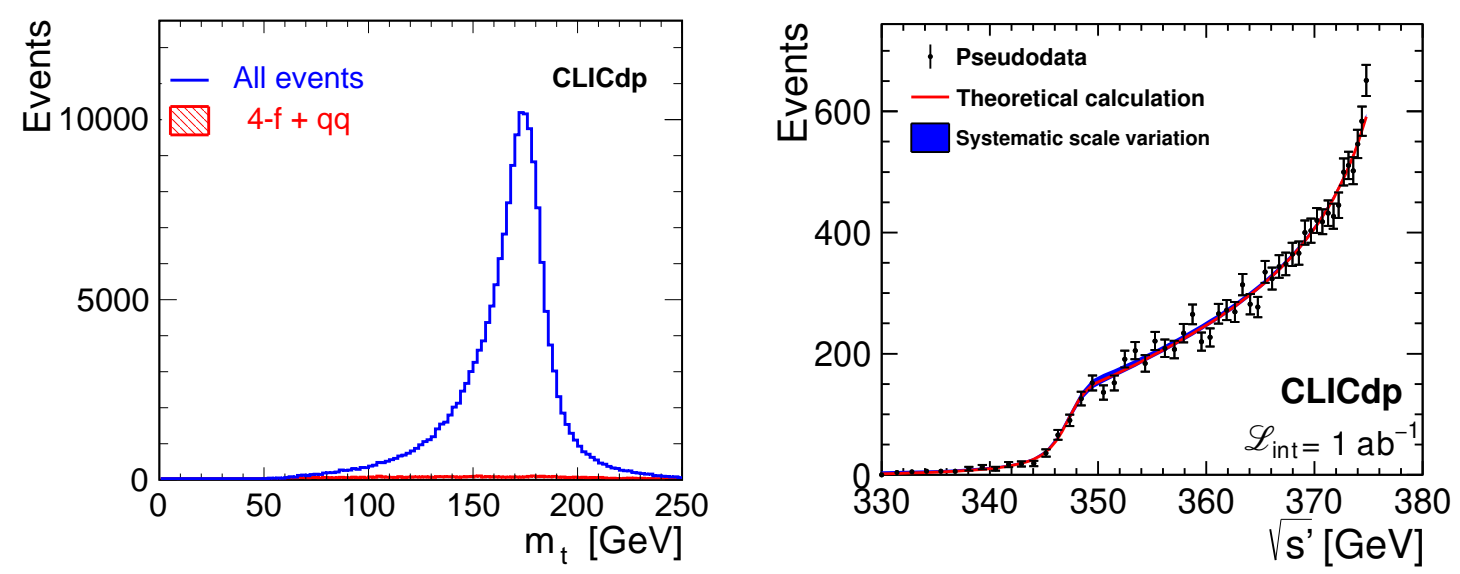

Figure 2: Distributions of the top-quark mass reconstructed from the hadronic top-quark decays in semi-

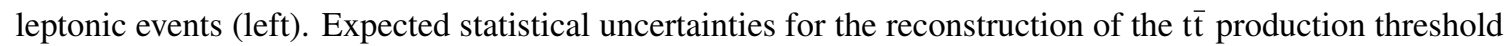
from the radiative event sample, $\mathrm{e}^{+} \mathrm{e}^{-} \rightarrow \mathrm{t} \overline{\mathrm{t}} \gamma$ (right). Figures for $1.0 \mathrm{ab}^{-1}$ collected at $380 \mathrm{GeV}$ CLIC [3].

ity spectrum [3]. However, the shape of the cross section as a function of collision energy has a strong dependence also on the top Yukawa coupling and the strength of the strong coupling. Dedicated analysis [5] indicates that, in order to extract the top-quark mass with a precision of the order of $20 \mathrm{MeV}$, the Yukawa coupling has to be constrained from other measurement to about $5 \%$ and the strong coupling constant has to be known to better than 0.0003, see Fig.1 (right). Combined theoretical uncertainties are estimated to be below $50 \mathrm{MeV}$.

The top-quark mass can also be extracted when running above the threshold. A statistical precision of around $30 \mathrm{MeV}$ is expected at the first CLIC stage where the top-quark mass is reconstructed from hadronic decay products, when combining hadronic and semi-leptonic pair production samples [3], see Fig.2 (left). Unfortunately, this measurement is a subject to significant theoretical uncertainties and also requires an excellent control of the jet energy scale to match the statistical accuracy. The top-quark mass can be also extracted from the cross section of radiative events, $\mathrm{e}^{+} \mathrm{e}^{-} \rightarrow \mathrm{t} \overline{\mathrm{t}} \gamma$, where a top-quark pair is produced in association with an energetic ISR photon radiated from the incoming electron or positron beam, see Fig.2 (right). With the assumed integrated luminosity of $1 \mathrm{ab}^{-1}$ collected at $380 \mathrm{GeV}$, a statistical precision of about $100 \mathrm{MeV}$ can be obtained and the total uncertainty is estimated to be around $140 \mathrm{MeV}$ [3].

\section{Precision top-quark measurements}

FCNC top-quark decays are very strongly suppressed in the SM, with expected branching ratios of the order of $10^{-14}$ to $10^{-12}$, while significant enhancement is expected in many BSM scenarios. Thanks to the high statistics of the produced top-quark pairs, precise final state reconstruction and very efficient flavour tagging, CLIC limits can be competitive with the expected HL-LHC reach for channels involving charm quarks.

The highest sensitivity to FCNC decays is expected at the first stage of CLIC. Candidate events are selected based on global event properties, jet clustering results, flavour tagging as well as lepton and photon identification and isolation requirements. Expected limits are then calculated using the 

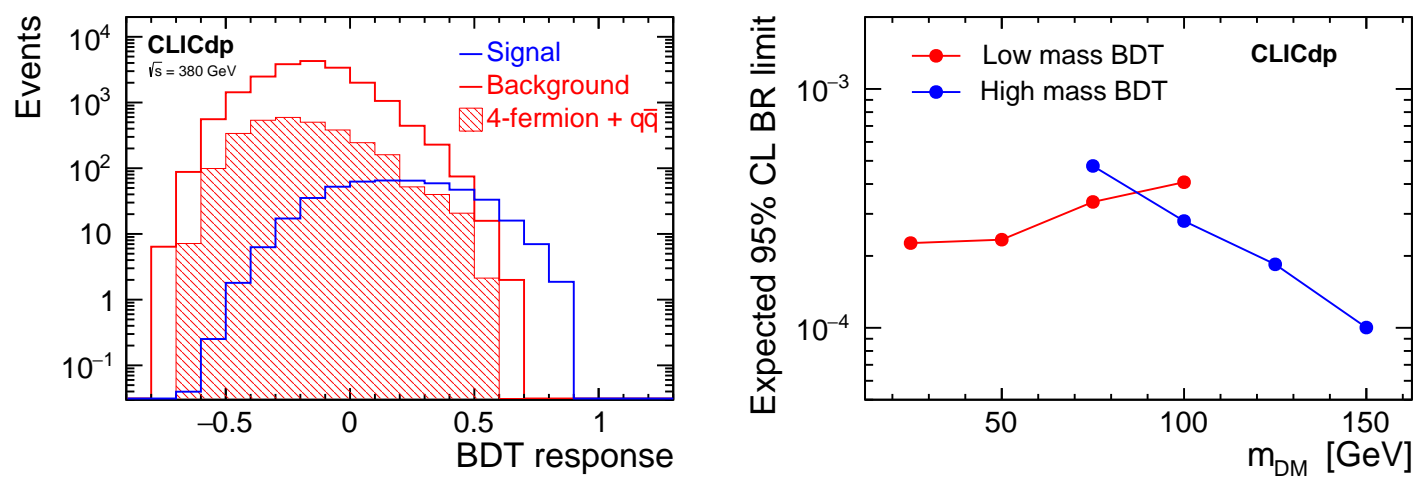

Figure 3: Distribution of the BDT classifier response for events with FCNC top-quark decays $\mathrm{t} \rightarrow \mathrm{cH}$ and SM background samples (left) and the expected 95\% C.L. limits on the top quark FCNC decay $\mathrm{t} \rightarrow \mathrm{cE}$ for $1 \mathrm{ab}^{-1}$ collected at $380 \mathrm{GeV}$ CLIC (right). Figures taken from [3].

$\mathrm{CL}_{\mathrm{s}}$ approach, based on the response distribution of the Boosted Decision Tree (BDT) classifier trained to discriminate between signal and background events. Results of the kinematic fits for signal and background hypotheses are included as input variables. An example distribution of the BDT classifier response for events with FCNC top-quark decays $\mathrm{t} \rightarrow \mathrm{c} \gamma$ and SM background samples is presented in figure 3 (left). Expected 95\% C.L. limits for $1 \mathrm{ab}^{-1}$ collected at $380 \mathrm{GeV}$ CLIC are: $\mathrm{BR}(\mathrm{t} \rightarrow \mathrm{c} \gamma)<2.6 \cdot 10^{-5}, \mathrm{BR}(\mathrm{t} \rightarrow \mathrm{cH}) \times \mathrm{BR}(\mathrm{H} \rightarrow \mathrm{b} \overline{\mathrm{b}})<8.8 \cdot 10^{-5}$ and $\mathrm{BR}(\mathrm{t} \rightarrow \mathrm{cE})<$ $1.0-3.4 \cdot 10^{-4}$, see figure 3 (right).

At the higher CLIC energy stages, the top Yukawa coupling can be directly obtained from the measurement of the $\mathrm{e}^{+} \mathrm{e}^{-} \rightarrow \mathrm{t} \overline{\mathrm{t}} \mathrm{H}$ cross section. ${ }^{1}$ Both fully-hadronic and semi-leptonic top-quark pair decays are considered, leading to final states with eight or six jets, respectively, including four b-quark jets. Distribution of the BDT classifier response used for the semi-leptonic analysis is compared for signal and background events in Fig.4 (left) [6]. The relative statistical precision of the Yukawa coupling determination is $2.7 \%$, assuming $2.5 \mathrm{ab}^{-1}$ of data collected at $1.5 \mathrm{TeV}\left(2 \mathrm{ab}^{-1}\right.$ with $-80 \%$ electron beam polarisation and $0.5 \mathrm{ab}^{-1}$ with $+80 \%$ electron beam polarisation) [3].

The high-energy stages of CLIC also allow the study of top-quark pair production in the vector boson fusion (VBF) process, $\mathrm{e}^{+} \mathrm{e}^{-} \rightarrow \mathrm{t} \overline{\mathrm{v}} v \bar{v}$. This production mode is particularly interesting because it is expected to be very sensitive to BSM effects. Shown in Fig.4 (right) is the distribution of the top-quark pair invariant mass for $\mathrm{e}^{+} \mathrm{e}^{-} \rightarrow \mathrm{t} \overline{\mathrm{t}} \mathrm{v} \overline{\mathrm{v}}$ events selected at $3 \mathrm{TeV}$ CLIC. Also indicated is the expected modification of the distribution due to BSM contributions described by the EFT operator $\mathrm{Q}_{\phi \mathrm{t}}[3]$.

\section{Constraining BSM scenarios}

Precision top quark measurements at CLIC are sensitive to many BSM scenarios. New physics effects can be constrained through measurements of the total pair-production cross-section, forwardbackward asymmetry as well as the helicity angle distribution in top decays. With high statistics of the top-quark pairs produced, these measurements can be made with percent level precision, see

\footnotetext{
${ }^{1}$ In the study presented here, the previous baseline assuming $1.4 \mathrm{TeV}$ for the second stage of CLIC is used.
} 

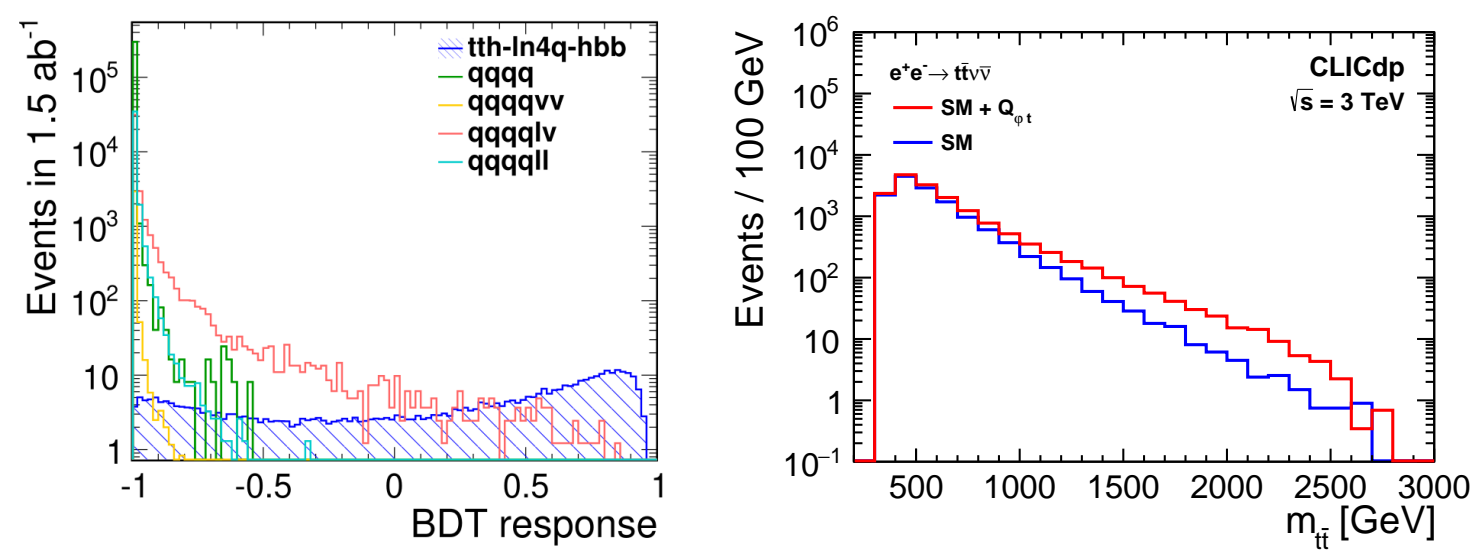

Figure 4: Distribution of the BDT classifier response for $\mathrm{e}^{+} \mathrm{e}^{-} \rightarrow \mathrm{t} \overline{\mathrm{t}} \mathrm{H}$ signal events and the considered background samples at $1.4 \mathrm{TeV}$ CLIC, for the semi-leptonic channel analysis (left) [6]. Distribution of the top-quark pair invariant mass for $\mathrm{e}^{+} \mathrm{e}^{-} \rightarrow \mathrm{t} \overline{\mathrm{t}} v \bar{v}$ events (VBF) at $3 \mathrm{TeV}$ CLIC, after all selection cuts, illustrating the possible effects due to the EFT operator $\mathrm{Q}_{\phi t}$ (right) [3].

Fig.5 (left). Additional constraints result from combining measurements performed with different electron beam polarisations and at different collision energies. Analysis of the top quark measurements is performed in the general framework of the SM-like Effective Field Theory (EFT). When data collected at different energy stages are combined, seven top-philic EFT operators can be constrained simultaneously from the global data fit, as shown in Fig.5 (right). Operation at high energy dramatically improves the sensitivity to the four-fermion operators and the limits set on the Wilson coefficients correspond to the new physics mass scales approaching $100 \mathrm{TeV}$.

The results from the global EFT analysis can also be interpreted in the framework of the topquark compositeness models. Figure 6 compares the expected CLIC $5 \sigma$ discovery regions for the analysis of observables related to $t \bar{t}$ and $t \bar{t} H$ production.
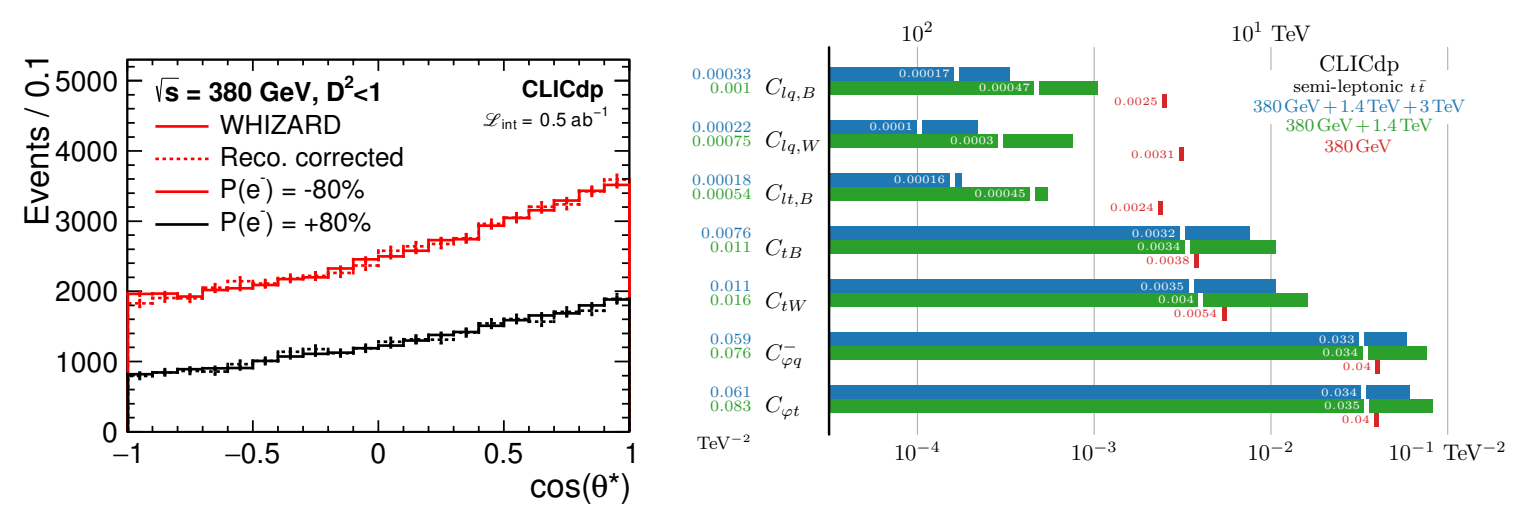

Figure 5: Top-quark polar angle distributions for operation at $\sqrt{s}=380 \mathrm{GeV}$ after the application of a tight quality cut (left) and the expected limits on the Wilson coefficients from the global EFT analysis of top-quark pair production at the $380 \mathrm{GeV}$ (right). Figures taken from [3]. 

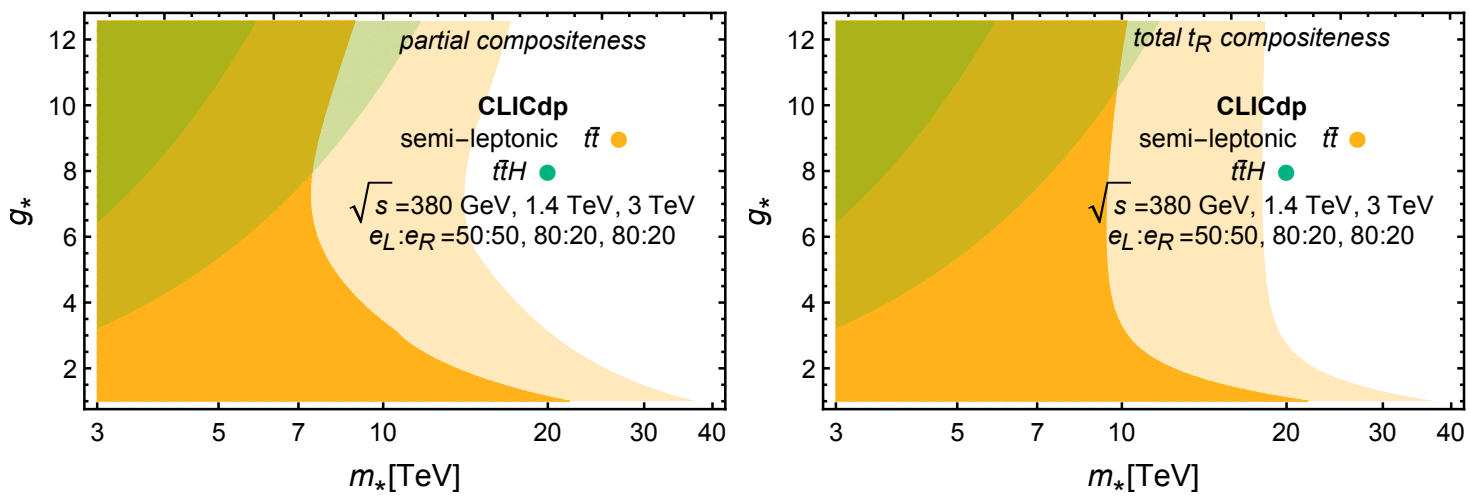

Figure 6: Expected $5 \sigma$ discovery regions of CLIC for the partial top-quark compositeness (left) and the total $t_{R}$ compositeness (right) scenarios. The orange contours were derived from the global fit to the $t \overline{\mathrm{t}}$ production observables, while the green contours were derived from the top Yukawa analysis. "Optimistic" (light color) and "pessimistic" (dark color) exclusion limits are compared, corresponding to minimal and maximal likelihood at each point of the $\left(m_{\star}, g_{\star}\right)$ plane, when varying other model parameters. Figures taken from [3].

\section{Conclusions}

A precise determination of top quark properties is crucial for the validation of the Standard Model or for pointing to an alternative BSM theory. A detailed study of the top-quark physics reach of CLIC has been presented in [3] and summarised in this contribution. The assumed CLIC staging scenario is well suited for top physics. High precision and background suppression capabilities of the CLIC detector will allow per mille level measurements and searches for rare processes. Sensitivity to BSM effects in the top-quark sector extends to the $100 \mathrm{TeV}$ scale.

\section{References}

[1] M. Aicheler et al., A Multi-TeV Linear Collider Based on CLIC Technology : CLIC Conceptual Design Report, CERN-2012-007 (2012).

[2] M. J. Boland et al. [CLIC and CLICdp Collaborations], Updated baseline for a staged Compact Linear Collider, CERN-2016-004 [arXiv: 1608 .07537].

[3] H. Abramowicz et al. [CLICdp Collaboration], Top-Quark Physics at the CLIC Electron-Positron Linear Collider, accepted for publication in JHEP, CLICdp-Pub-2018-003, [arXiv: 1807.02441 ].

[4] A. Robson and P. Roloff, Updated CLIC luminosity staging baseline and Higgs coupling prospects, [arXiv:1812.01644].

[5] K. Nowak and A.F. Żarnecki, Extracting the top-quark mass and Yukawa coupling from the threshold scan at CLIC, Proc. SPIE 11176, Photonics Applications in Astronomy, Communications, Industry, and High-Energy Physics Experiments 2019, 1117648 (2019).

[6] S. Redford, P. Roloff and M. Vogel, Study of the effect of additional background channels on the top Yukawa coupling measurement at a 1.4 TeV CLIC, CLICdp-Note-2015-001 (2015). 